\title{
ANÁLISE DE MEDIDAS DE SISTEMAS COM MÚLTIPLAS ENTRADAS E MÚLTIPLAS SAÍDAS NA FREQUÊNCIA DE 2,4 GHZ EM AMBIENTES INTERNOS
}

Luiz Carlos F. Nogueira

Julio Cesar R. Dal Bello

\begin{abstract}
Resumo: Um novo e importante sistema de acesso sem fio, que vem sendo implementado em modernos sistemas de telecomunicações, utiliza múltiplas antenas transmissoras e múltiplas antenas receptoras. Através deste sistema, conhecido como Sistema MIMO (Multiple-Input Multiple-Output System), é possível melhorar a capacidade e a área de cobertura, tornando o acesso sem fio mais competitivo em relação ao acesso cabeado, em termos de taxa de transmissão. Neste trabalho será apresentado um setup inédito para realização de medidas de sistemas MIMO, na frequência de $2,4 \mathrm{GHz}$, utilizando a tecnologia WiFi, bem como serão descritas as campanhas de medidas realizadas em ambientes internos típicos, com análises das mesmas para indicar os benefícios que podem ser alcançados com tais sistemas.
\end{abstract}

Palavras chave: MIMO, sistemas MIMO, tecnologia MIMO, propagação.

Abstract: A new and important access system that has been implemented in modern wireless systems uses multiples transmitting antennas and multiples receiving antennas. Through this system, known as MIMO (Multiple-Input Multiple-Output System), it is possible to achieve benefits in capacity and coverage area, and let the wireless system better in comparison to the traditional wired systems in terms of data transmission throughput. To achieve and optimize these benefits it is necessary to know some parameters that will be analyzed on this work. Besides, in this work it is shown a new MIMO setup system in $2.4 \mathrm{GHz}$, using WiFi technology, as well as measurement campaigns performed on typical indoor and outdoor places. Analyses are done to check the benefits to be acquired with these systems.

Keywords: MIMO, MIMO Systems, MIMO Technology, radiowave propagation

\section{INTRODUÇÃO}

Neste artigo é feita uma análise dos benefícios que podem ser alcançados através de um sistema que opera com múltiplas antenas transmissoras e múltiplas antenas receptoras. A partir de experimentos realizados no Campus Praia Vermelha da Universidade Federal Fluminense, em ambientes internos (indoor), foi construído um banco de dados e feitas análises estatísticas que comprovam o ganho relativo em função das múltiplas antenas, corroborando o potencial da tecnologia MIMO para uso em sistemas práticos.
No Brasil, atualmente está sendo implantada a terceira geração de sistemas celulares (3G) e os sistemas WiMAX, entre outros sistemas, com o objetivo de aumentar a capacidade de transferência de dados. Para tanto, os sistemas sem fio modernos utilizam uma série de novas tecnologias, sendo a tecnologia MIMO a que se destaca. Neste artigo, inicialmente é feita uma abordagem teórica básica da tecnologia MIMO e, em seguida, uma análise dos resultados das medidas realizadas que comprovam os benefícios que podem ser alcançados com esta tecnologia.

\footnotetext{
${ }^{1}$ Luiz Carlos Figueira Nogueira, Unidade Descentralizada de Nova Iguaçu, Centro Federal de Educação Tecnológica Celso Suckow da Fonseca, Nova Iguaçu, RJ, Brasil, E-mail: lctelecom@gmail.com.

Julio Cesar R. Dal Bello, Departamento de Engenharia de Telecomunicaçôes, Universidade Federal Fluminense, Niterói, Brasil E-mail: dalbello@telecom.uff.br.
} 


\section{CONCEITOS BÁSICOS}

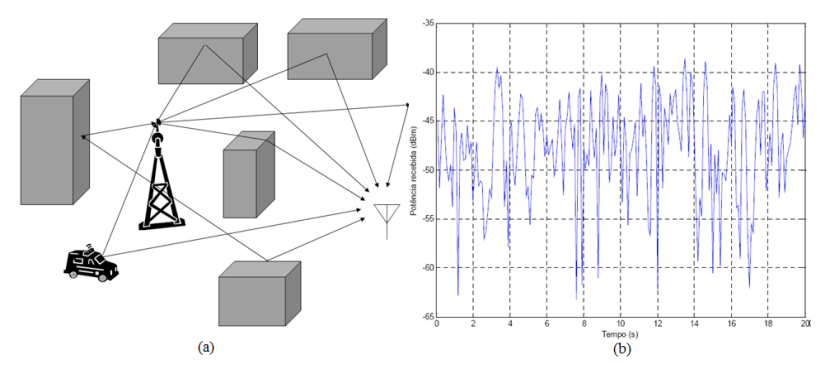

Fig. 1 - (a) Ambiente típico de radiopropagação.

(b) Desvanecimento em pequena escala: potência recebida, em $\mathrm{dBm}$, num ambiente sem visada, na frequência de $1 \mathrm{GHz}$, com Doppler máximo de $5 \mathrm{~Hz}$

O sinal recebido num sistema de comunicações sem fio está associado à resposta do canal rádio-móvel em função dos múltiplos percursos das ondas que se propagam entre o transmissor e o receptor. Estes multipercursos funcionam como uma "série de ecos" [1] e [2] devido a reflexôes, refraçôes, difraçōes e espalhamento no ambiente de propagação. Além da possibilidade de movimentação do receptor e/ou transmissor, normalmente existem pessoas, veículos etc. se deslocando no ambiente, de forma que são geradas oscilaçôes aleatórias na intensidade do sinal recebido, fenômeno conhecido como desvanecimento [1], conforme mostra a figura 1 (a) e (b), para o caso de um ambiente típico de radiopropagação.
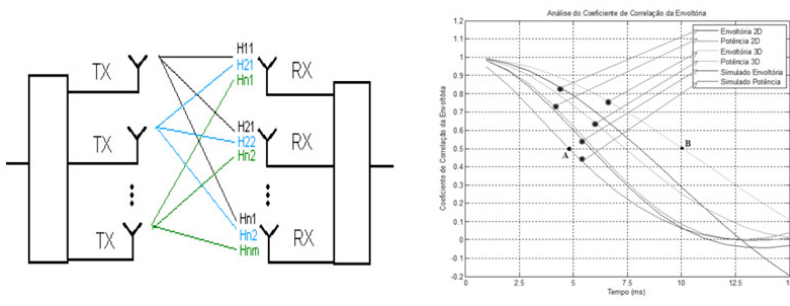

Fig. 2 - (a) Sistema MIMO com caracterização da matriz do canal. (b) Análise do coeficiente de correlação para a frequência de $3 \mathrm{GHz}$ e doppler de $30 \mathrm{~Hz}$ Ambiente típico de radiopropagação

O sistema MIMO é constituído por múltiplas antenas na transmissão e na recepção, formando vários canais [3] e [4], conforme está indicado na figura 2 (a), onde $\mathrm{Hi}, \mathrm{j}(\mathrm{i}=1, . ., \mathrm{n}$; $\mathrm{j}=1, \ldots, \mathrm{m})$ é a matriz de ganho normalizado do canal. Assim sendo, os múltiplos canais do sistema MIMO diminuem o efeito do desvanecimento seletivo, porém para garantir a eficiência no combate ao desvanecimento é necessário posicionar as antenas transmissoras e receptoras de forma que os sinais cheguem descorrelacionados, ou seja, o coeficiente de correlação do sinal recebido seja menor que 0,5 . Esta é uma situação estatística favorável para que se evite a queda do nível do sinal recebido de forma simultânea nas diversas antenas receptoras. A figura 2 (b) mostra as curvas do coeficiente de correlação do sinal recebido, em função da envoltória e da potência (em duas e três dimensões), obtidas em modelos matemáticos teóricos desenvolvidos, e em ambiente simulado através de medidas adquiridas num ambiente real, para a frequência de $3 \mathrm{GHz}$ e Doppler de $30 \mathrm{~Hz}$.

Analisando a figura 2 (b), no ponto A verifica-se que em $5 \mathrm{~ms}$ o modelo simulado está descorrelacionado, o que corresponde a uma distância de $0,015 \mathrm{~m}(1,5 \lambda)$, e que no ponto $\mathrm{B}$ a descorrelação ocorre em $10 \mathrm{~ms}$ (pior caso do modelo teórico), de forma que corresponde a

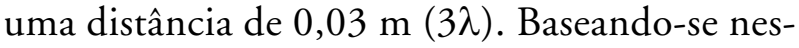
tas análises, e em outras pesquisas desenvolvidas com o mesmo foco, normalmente adota-se que para distâncias maiores que 3 vezes o comprimento de onda $(3 \lambda)$ o sinal estará descorrelacionado.

Um caso específico em que o sistema MIMO pode não apresentar eficiência é quando há "gargalos" simples e múltiplos, casos conhecidos na literatura internacional como keyholes. Nestes casos, o gargalo cria um percurso, ou mais, em que todos os canais podem sofrer atenuação simultânea, independentemente da quantidade de antenas, conforme indica a figura 3 .

\section{EQUIPAMENTOS DE MEDIDAS (SETUP)}

Para possibilitar a realização dos testes de propagação necessários aos estudos, foi desenvolvido e configurado um setup inédito, que passa a ser uma contribuição adicional deste trabalho para o estudo dos canais MIMO, pelas suas características intrínsecas de praticidade e simplicidade de operação [5]. Os equipamentos que compõem o setup serão descritos a seguir. 


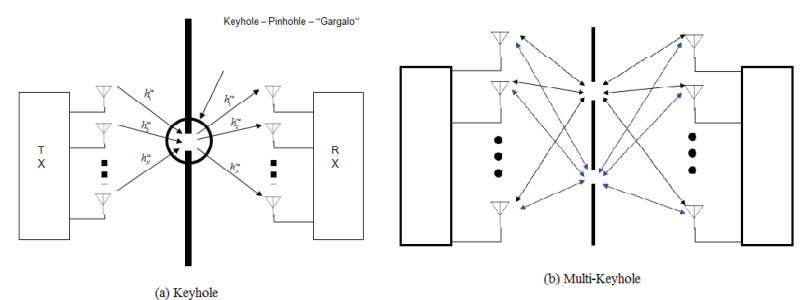

Fig. 3. Limitações para desempenho do sistema MIMO: (a) Keyhole simples; (b) Múltiplos Keyhole.

Fig 3 - Limitações para desempenho do sistema MIMO: (a) Keyhole simples; (b) Múltiplos Keyhole

\section{A - EQUIPAMENTO DE TRANSMISSÃO E RECEPÇÃO}

Para fazer as medidas necessárias para estudo do comportamento de um canal MIMO, foram utilizados dois transmissores Wi-Fi (IEEE 802.11). Na recepção, foram utilizados dois módulos com duas antenas setoriais, com abertura vertical e horizontal de 60 graus, com ganho de $12 \mathrm{dBi}$, conforme ilustra a figura 4 .

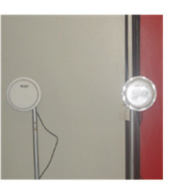

(a)

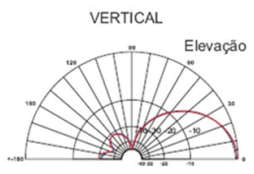

(b)

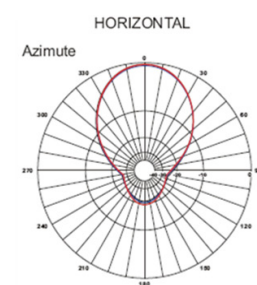

(c)
Fig. 4 - (a) Setup de Recepção: (a) Antenas receptoras, (b) diagrama de irradiação vertical das antenas e (c) diagrama de irradiação horizontal das antenas.

Para a aquisição dos dados, foram usadas duas placas Wi-Fi juntamente com o programa computacional Network Stumbler, versão 0.4.0, desenvolvido por Marius Milner [11]. O programa Network Stumbler possui a facilidade de possibilitar a exportação dos dados. A taxa de amostragem na recepção de cada sinal transmitido foi, em média, de duas amostras por segundo (resolução horizontal) com variação de -100 a 0 $\mathrm{dBm}$, de 2 em $2 \mathrm{~dB}$ (resolução vertical). O diagrama lógico do sistema de medidas está mostrado na figura 5.

A distância de separação entre as antenas que formam o par de transmissão (dipolos) e o par de recepção (antenas diretivas) foi mantida em $60 \mathrm{~cm}$, para garantir a descorrelação do sinal.

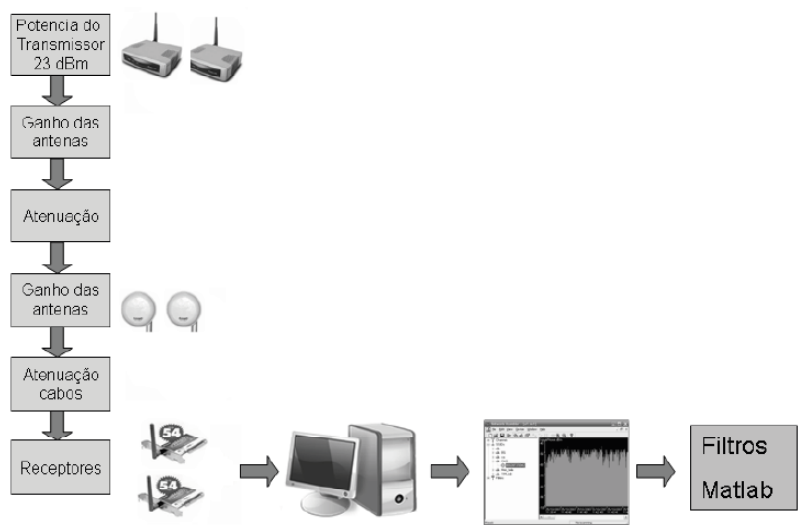

Fig. 5 - Diagrama lógico do sistema de medidas

\section{AMBIENTES DE MEDIÇÃO E ANÁLISE DOS RESULTADOS}

\section{A. MEDIDAS EM AMBIENTES INTERNOS E ANÁLISE DOS RESULTADOS}

A campanha de medições internas foi realizada nos meses de setembro e outubro de 2007, no quarto andar do bloco D do Campus Praia Vermelha da UFF, em horários onde havia circulação de pessoas. Neste ambiente há uma grande quantidade de espalhadores como pilastras, caixas metálicas de energia, infra-estrutura de cabeamento junto à parte superior, paredes de tijolo e mobília típica de área universitária [6] e [7]. As mediçóes internas foram realizadas no bloco $D$, nas 8 posições diferentes mostradas na figura 6 , cada uma com 4 enlaces, totalizando 32 enlaces e os resultados obtidos estão apresentados na Tabela 1. Neste artigo, será relatado e ilustrado apenas o procedimento adotado para a posição 2 , enlace 1 , que apresentou os resultados mostrados na figura 7 , e que foram recorrentes para todas as demais posições.

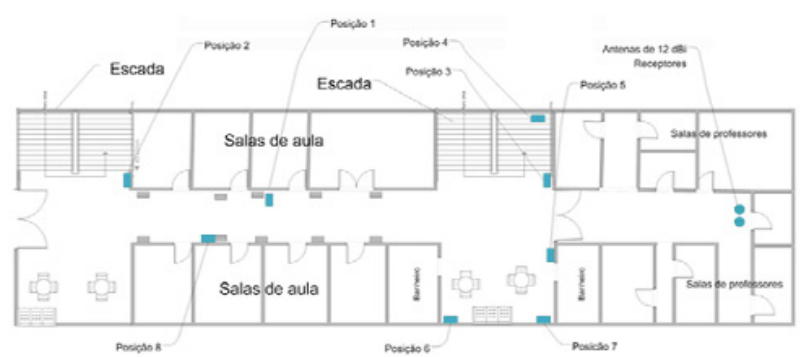

Fig. 6 - Ambientes internos de medidas - Bloco D do Campus Praia Vermelha a UFF, onde os pontos de medidas são indicados pelos blocos 

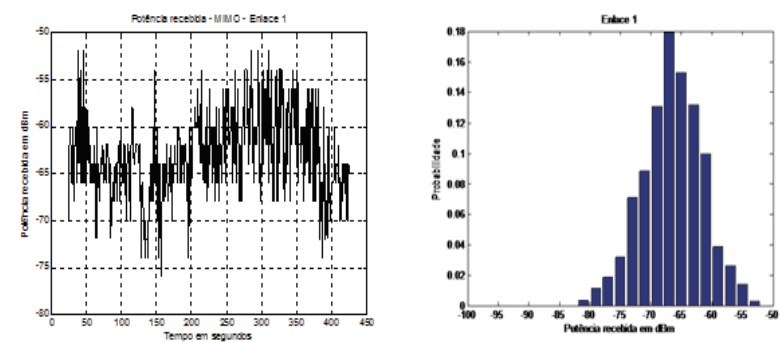

Fig. 7 - (a) Nível de potência recebida, em $\mathrm{dBm}$, na posição 2 (Enlace 1), numa janela de 450 segundos, (b) densidade de probabilidade do enlace, sendo no eixo vertical a probabilidade variando de 0 a 0.18 , com intervalos de 0.02 e no eixo horizontal a potência recebida em $\mathrm{dBm}$, variando de -100 a -50, com intervalos de 5

As mediçōes foram realizadas na condição de propagação onde não há visada direta entre as antenas (NLOS), e a distância entre os transmissores e os receptores foi mantida em 85 metros. A figura 7(a) mostra o comportamento do sinal recebido, em função do tempo, na posição 2 do enlace 1, e a figura 7 (b) mostra a função densidade de probabilidade (PDF) correspondente.

A função densidade de probabilidade (PDF) de cada enlace foi traçada a partir de um sistema de medição no qual a resolução vertical varia de 2 em $2 \mathrm{dBm}$, marcando-se somente os números pares. Assim sendo, quando se traça a PDF com os dados da tabela fornecida pelo setup de medição, a função ganha a forma discreta [1] e [8].

Uma análise importante a ser feita num Sistema MIMO é sobre o comportamento da diversidade. Entretanto, por causa das limitaçóes do setup utilizado, não foi possível fazer o teste matemático da correlação. Entretanto, selecionando-se o pior momento em relação ao desvanecimento, observou-se que os 4 canais da posição 2 não sofreram atenuação profunda de forma simultânea. O mesmo comportamento foi observado na grande maioria dos canais MIMO medidos, como também relatam diversos autores [9] a [16].

\section{B - ANÁLISE COMPARATIVA DOS RESULTADOS OBTIDOS}

Nas mediçóes internas realizadas no quarto andar do bloco D do Campus Praia Vermelha da UFF, foram aquisitadas mais de 30 mil amostras, e a diferença entre a média da potência recebida nos canais variou de 9,4 a $2 \mathrm{~dB}$, com uma média de 4,5
$\mathrm{dB}$ e desvio padrão de 2,6 dB. A diferença desses valores para o nível de cobertura de $90 \%$ do sinal recebido foi de 12 a $2,5 \mathrm{~dB}$, com média de 5,4 $\mathrm{dB}$ e desvio padrão de $3,6 \mathrm{~dB}$. O comportamento do nível médio do sinal recebido, em $\mathrm{dBm}$, nas 8 posiçōes internas, está mostrado na tabela 1 .

Na tabela 1 verifica-se que há um ganho na etapa de radiopropagação do Sistema MIMO, sem contar com o ganho que pode ser conseguido com os métodos de combinação na etapa de recepção. Consequentemente, é possível melhorar a capacidade do canal com os sistemas MIMO, além de mitigar o desvanecimento profundo.

Tabela 1 - Nível médio do sinal recebido, em $\mathrm{dBm}$, nas posições internas

\begin{tabular}{|c|c|c|c|c|c|c|c|c|}
\hline \multicolumn{9}{|c|}{ POSIÇÕES INTERNAS } \\
\hline & 1 & 2 & 3 & 4 & 5 & 6 & 7 & 8 \\
\hline Enlace & $\begin{array}{l}\text { Média } \\
(\mathrm{dBm})\end{array}$ & $\begin{array}{l}\text { Média } \\
(\mathrm{dBm})\end{array}$ & $\begin{array}{l}\text { Média } \\
\text { (dBm) }\end{array}$ & $\begin{array}{l}\text { Média } \\
(\mathrm{dBm})\end{array}$ & $\begin{array}{l}\text { Médıa } \\
\text { (dBm) }\end{array}$ & $\begin{array}{l}\text { Média } \\
\text { (dBm) }\end{array}$ & $\begin{array}{l}\text { Média } \\
\text { (dBm) }\end{array}$ & $\begin{array}{l}\text { Média } \\
\text { (dBm) }\end{array}$ \\
\hline 1 & $-46,7$ & $-65,4$ & $-52,3$ & $-65,4$ & $-54,6$ & $-62,9$ & $-66,8$ & $-65,5$ \\
\hline 2 & -46 & $-65,8$ & $-55,2$ & $-65,8$ & $-55,7$ & $-66,4$ & $-67,9$ & $-67,2$ \\
\hline 3 & $-53,1$ & $-67,4$ & $-58,1$ & $-67,4$ & $-52,3$ & -65 & -70.5 & $-61,9$ \\
\hline 4 & $-55,5$ & $-67,1$ & $.59,3$ & $-67,1$ & $-52,1$ & $.65,2$ & $.70,2$ & $-63,3$ \\
\hline Difereaşa (dB) & 9,4 & 1,9 & 7,0 & 1,9 & 3,5 & 3,5 & 3,7 & 5,24 \\
\hline $\begin{array}{l}\text { Modiz des } \\
\text { Diforenças }\end{array}$ & \multicolumn{8}{|c|}{$4,6(d B)$} \\
\hline $\begin{array}{l}\text { Desvio Padräo } \\
\text { das Diferenças }\end{array}$ & \multicolumn{8}{|c|}{$2,6(\mathbb{C B})$} \\
\hline
\end{tabular}

Esses resultados permitem explorar um novo conceito em projetos de cobertura de sistemas MIMO, que teriam 3 regiôes: região de alta capacidade de transmissão, região de transição e região de utilização de sistemas típicos de ganho por diversidade, com combinação dos sinais, conforme mostra a figura 8 .

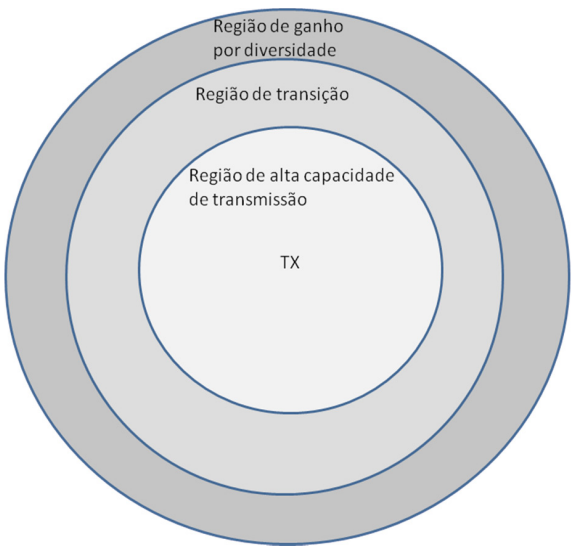

Fig. 8 - Célula MIMO típica com 3 regiōes. 


\section{CONCLUSÓES}

O elemento mais importante num sistema de comunicaçôes sem fio é o sinal recebido, pois é através deste que se verifica se há condições para que ocorra a transmissão de dados de maneira eficaz. O nível de ruído é importante para se determinar a capacidade do canal, porém, no caso deste artigo, a potência de ruído esteve sempre abaixo da potência mínima admitida pelo setup de recepção e, consequentemente, não foi detectada e registrada. De qualquer forma, o foco deste artigo é a análise da potência recebida (de sinal), em função do ambiente de propagação com múltiplos canais.

De acordo com as diversas análises em função dos resultados obtidos com as mediçôes realizadas no Campus da Praia Vermelha da UFF, percebeu-se que grandes benefícios ocorrem com a utilização do sistema MIMO para diminuir os efeitos do desvanecimento rápido. A teoria de canais MIMO, que foi sinteticamente tratada neste trabalho, foi simulada e comprovada através das medidas dos canais em ambientes internos, utilizando-se um setup inédito em função de sua praticidade e facilidade de operação.

Fica evidente a maior confiabilidade dos sistemas MIMO em relação ao desvanecimento rápido, em função da diversidade de canais, pois não houve momento em que os quatro canais sofressem desvanecimento profundo de forma simultânea, conforme indicam as análises das medições realizadas. Além disso, um aumento significativo pode ser alcançado nas taxas de transmissão em função da utilização de múltiplos canais.

Uma característica importante observada, através das medições, é que alguns canais possuíam o valor do sinal recebido muito maior do que os recebidos nos outros canais. Assim, a separação das antenas garante um relativo ganho em relação ao valor médio do sinal recebido.

Há expectativa de utilização do sistema MIMO em novos padrões do IEEE, como o padrão IEEE 802.11n, nos sistemas WIMAX (IEEE 802.16), em sistemas 3G (UMTS) e, mais amplamente, nos futuros sistemas $4 \mathrm{G}$.

\section{REFERÊNCIAS}

[1] R VAUGHAN E J B ANDERSEN, "Channel, Propagation and Antennas for Mobile Com- munications", Livro, The Institution of Electrical Engineers, London, United Kingdom, 2003.

[2] W C Y LEE, "Mobile Communications Engineering", Livro, McGraw-Hill Telecommunications, 1998.

[3] G J FOSCHINI E M J GAN, "On Limits of Wireless Communications in a Fading Environment when Using Multiple Antennas"; Artigo, Wireless Personal Commun, Vol. 6, pp. 311-335, Mar. 1998B.

[4] R D VIEIRA, "Medidas do Canal MIMO Indoor: Analise da Capacidade do Canal e dos Parâmetros do Canal”, Tese de Doutorado, PUC Rio.

[5] L C F NOGUEIRA, "Análise de Medidas de Sistemas MIMO na Frequência de 2,4 GHz em Ambientes Internos e Externos", Dissertação de Mestrado, UFF, 2008.

[6] J C R DAL BELLO, “Caracterização da Influência da Vegetação nos Sistemas de Comunicaçôes Móveis Celulares em Áreas Urbanas", Tese de Doutorado, PUC Rio.

[7] L J MATOS, "Influência da Vegetação na Dispersão dos Sinais Rádio-Móveis”, Tese de Doutorado, PUC Rio, 2005

[8] A PAPOULIS, "Probability, Random Variables, and Stochastic Processes", Livro, McGrawHill Series in Electrical Engineering, 2002

[9] FOSCHINI, G.J.; GANS M.J.; “On Limits of Wireless Communications in a Fading Environment when Using Multiple Antennas"; Artigo, Wireless Personal Commun, Vol. 6, pp. 311335, Mar. 1998

[10] MARZETTA, T. L.; HOCHWALD, B. M., "Capacity of a mobile multiple antenna communication link in flat fading", IEEE Transaction on Information Theory, Vol. 45, pp. 139-157, January 1999

[11] AL-DHAHIR, N.; "Finite-Length MIMO Decision Feedback Equalization for Space-Time Block-Coded Signals over Multipath-Fading Channels", Artigo, IEEE Transactions on Vehi- 
cular Technology, vol. 50, no. 4, pp.1176-1182, 2001

[12] WALLACE, J.; JENSEN, M.; "Measured Characteristics of the MIMO Wireless Channel"; Artigo, Department of Electrical and Computer Engineering Brigham Young University, Provo,; 2001

[13] THOMA, R.S.; HAMPICKE, D.; RICHTER, A., SOMMERKORN, G., "MIMO Vector Channel Sounder Measurement for Smart Antenna System Evaluation, European Transactions on Telecommunications", ETT, Vol. 12, No.5, pp. 427-438, 2001

[14] KERMOAL, J. P.; "A Stochastic MIMO Radio Channel Model With Experimental Validation";
Artigo, IEEE Journal on Selected Areas in Communications, vol. 20, no. 6,, pp.1211-1226, 2002

[15] VALENZUELA, REINALDO A. ; CHUAH, CHEN-NEE; TSE, DAVID N. C.; KAHN, JOSEPH M.; "Capacity Scaling in MIMO Wireless Systems Under Correlated Fading “, Artigo, IEEE TRANSACTIONS ON INFORMATION THEORY, VOL. 48, NO. 3, MARCH 2002

[16] D. GESBERT, H. BOLCSKEI, D. GORE, AND A. PAULRAJ, "Outdoor MIMO wireless channels: models and performance prediction” IEEE Trans. Commun., vol. 50, no. 12, pp. 1926-1934, 2002 